\title{
Role of mTOR signaling in the regulation of high glucose-induced podocyte injury
}

\author{
QIUYUE LI, YAN ZENG, QING JIANG, CONG WU and JING ZHOU \\ Nephrology Department, The First Affiliated Hospital of Nanchang University, Nanchang, Jiangxi 330006, P.R. China
}

Received May 16, 2018; Accepted December 12, 2018

DOI: $10.3892 /$ etm.2019.7236

\begin{abstract}
Podocyte injury, which promotes progressive nephropathy, is considered a key factor in the progression of diabetic nephropathy. The mammalian target of rapamycin (mTOR) signaling cascade controls cell growth, survival and metabolism. The present study investigated the role of mTOR signaling in regulating high glucose (HG)-induced podocyte injury. MTT assay and flow cytometry assay results indicated that HG significantly increased podocyte viability and apoptosis. HG effects on podocytes were suppressed by mTOR complex 1 (mTORC1) inhibitor, rapamycin, and further suppressed by dual mTORC1 and mTORC2 inhibitor, KU0063794, when compared with podocytes that received mannitol treatment. In addition, western blot analysis revealed that the expression levels of Thr-389-phosphorylated p70S6 kinase (p-p70S6K) and phosphorylated Akt (p-Akt) were significantly increased by HG when compared with mannitol treatment. Notably, rapamycin significantly inhibited HG-induced p-p70S6K expression, but did not significantly impact p-Akt expression. However, KU0063794 significantly inhibited the HG-induced p-p70S6K and p-Akt expression levels. Furthermore, the expression of ezrin was significantly reduced by HG when compared with mannitol treatment; however, $\alpha$-smooth muscle actin ( $\alpha$-SMA) expression was significantly increased. Immunofluorescence analysis on ezrin and $\alpha$-SMA supported the results of western blot analysis. KU0063794, but not rapamycin, suppressed the effect of $\mathrm{HG}$ on the expression levels of ezrin and $\alpha$-SMA. Thus, it was suggested that the increased activation of mTOR signaling mediated HG-induced podocyte injury. In addition, the present findings suggest that the mTORC1 and mTORC2 signaling pathways may be responsible for the cell viability and apoptosis, and that the mTORC2 pathway could be primarily responsible for the regulation of cytoskeleton-associated proteins.
\end{abstract}

Correspondence to: Mr. Jing Zhou, Nephrology Department, The First Affiliated Hospital of Nanchang University, 17 Yongwaizheng Street, Nanchang, Jiangxi 330006, P.R. China

E-mail: jingz6457@163.com

Key words: mammalian target of rapamycin complex 1, mammalian target of rapamycin complex 2, podocyte injury, high glucose, diabetic nephropathy

\section{Introduction}

Diabetic nephropathy (DN) is a common complication of diabetes that can promote the development of renal diseases (1). Patients with DN usually exhibit reduced filtration rates, albuminuria and ultimately renal failure (2). Multiple mechanisms have been implicated in the development and outcome of DN, including changes in hyperglycemia-induced metabolism, changes in hemodynamics and genetic predisposition (3). Patients with diabetes usually still develop massive and treatment-resistant proteinuria that can cause a rapid decline in renal function (4). Thus, further understanding of the pathogenesis in DN may help to improve renal and health outcomes in patients with diabetes.

Podocyte injury is a key event in the progression of DN that can induce the production of proteinuria and further cause the development of diabetic kidney disease (5). Podocytes have a limited ability to regenerate, thus the extent of podocyte injury is commonly regarded as an important prognostic determinant in DN (6). High glucose (HG) can result in glomerular injury, further induce chronic renal function loss and ultimately lead to the occurrence of end-stage renal disease (7-9). Previous studies have revealed that podocyte injury is an important early event leading to glomerular disease (10) in patients with DN $(11,12)$. However, the underling mechanisms involved in HG-induced podocyte injury remain unclear.

The mammalian target of rapamycin (mTOR), a serine/threonine kinase of the phosphoinositide 3-kinase-related kinase family, has been identified as the target of rapamycin (sirolimus) in mammals (13). mTOR is the core component of two distinct complexes complex 1 (mTORC1) and complex 2 (mTORC2) (14). As mTOR is specifically inhibited by rapamycin only when it is in mTORC1, mTORC1 has been initially defined as 'rapamycin sensitive', whereas mTORC2 has been defined as 'rapamycin insensitive' (15). The level of mTOR activity is associated with tubular cell proliferation (16), apoptosis (17-19) and autophagy (20). In has been revealed that the $\mathrm{mTORC} 2 / \mathrm{Akt} /$ nuclear factor- $\kappa \mathrm{B}$ signaling pathway can mediate the activation of transient receptor potential cation channel 6, which is involved in ADR-induced podocyte apoptosis (21). Inhibition of mTORC2 promotes the prevention of reactive oxygen species-induced apoptosis (22) and activation of mTORC1, which induces the expression of endoplasmic reticulum stress signaling and thus leads to apoptosis in HG-treated podocytes (23). In addition, dual targeting 
of mTORC1 and mTORC2 can promote the induction of autophagy in acute myeloid leukemia cells (24).

In the current study, it was hypothesized that the mTOR signaling pathway was involved in the regulation of HG-induced podocyte injury. Podocyte viability and apoptosis $24 \mathrm{~h}$ following $\mathrm{HG}$ treatment were assessed. In addition, the expression levels of mTOR signaling proteins and cytoskeleton-associated proteins were examined.

\section{Materials and methods}

Cell culture. Mouse podocytes (MPC5, provided by Professor Peter Mundel, Mount Sinai School of Medicine, New York, NY, USA) were cultured in RPMI 1640 medium (Gibco; Thermo Fisher Scientific, Inc., Waltham, MA, USA) supplemented with $10 \%$ heat-inactivated fetal calf serum (Gibco; Thermo Fisher Scientific, Inc.), $100 \mathrm{U} / \mathrm{ml}$ penicillin (Gibco; Thermo Fisher Scientific, Inc.) and $100 \mu \mathrm{g} / \mathrm{ml}$ streptomycin (Gibco; Thermo Fisher Scientific, Inc.) in a humidified atmosphere containing $5 \% \mathrm{CO}_{2}$. Cells were grown in RPMI 1640 medium containing $100 \mathrm{U} / \mathrm{ml}$ mouse interferon (IFN- $\gamma$; Peprotech EC Ltd., London, UK) at $33^{\circ} \mathrm{C}$ with $100 \%$ relative humidity and $5 \% \mathrm{CO}_{2}$, and were induced to differentiate at $37^{\circ} \mathrm{C}$ in a medium without IFN- $\gamma$ for $10-14$ days. Following differentiation for 10-14 days, the cells were subsequently divided into five groups: Normal glucose (NG; $5.6 \mathrm{mmol} / \mathrm{l}$ glucose), mannitol (M; $5.6 \mathrm{mmol} / 1$ glucose $+24.4 \mathrm{mmol} / \mathrm{l})$, $\mathrm{HG}$ (30 mmol/l glucose), $\mathrm{HG}+$ rapamycin (30 mmol/l glucose +50 nmol/1 rapamycin; MedChem Express, Monmouth Junction, NJ, USA) and HG + KU0063794 (30 mmol/1 glucose +2 $\mu \mathrm{mol} / 1$ KU0063794; MCE, NJ, USA) groups. The concentration of rapamycin or KU0063794 used was as described in previous studies $(25,26)$. Cells were exposed to different conditions for $24 \mathrm{~h}$ and then harvested for further experiments.

Cell viability assessment. Podocytes were seeded on a 96-well plate overnight at $2 \times 10^{4}$ cells/well and were subsequently incubated with $\mathrm{NG}$ (5.6 $\mathrm{mmol} / 1$ glucose), M (5.6 mmol/1 glucose + $24.4 \mathrm{mmol} / \mathrm{l}), \mathrm{HG}$ (30 mmol/1 glucose), $\mathrm{HG}+$ rapamycin (30 $\mathrm{mmol} / 1$ glucose $+50 \mathrm{nmol} / 1 \mathrm{rapamycin})$ or $\mathrm{HG}+\mathrm{KU} 0063794$ (30 mmol/l glucose $+2 \mu \mathrm{mol} / 1 \mathrm{KU} 0063794)$ for $24 \mathrm{~h}$. Thereafter, cell viability was measured using the MTT assay (Sigma-Aldrich; Merck KGaA, Darmstadt, Germany), according to the manufacturer's instructions. Briefly, a total of $10 \mu \mathrm{l}$ MTT $(5 \mathrm{mg} / \mathrm{ml})$ was added into each well and then incubated for $4 \mathrm{~h}$ at $37^{\circ} \mathrm{C}$. Next, $150 \mu \mathrm{l}$ dimethyl sulfoxide was added to the 96 -well plates. Finally, the absorbance was measured at $568 \mathrm{~nm}$ on a microplate reader. Results were calculated as percentages of the NG group (100\%).

Annexin V-fluorescein isothiocyanate (FITC)/propidium iodide (PI) staining. Podocytes were harvested via trypsinization and washed twice with cold phosphate buffered saline (PBS). Cells were centrifuged at $750 \mathrm{x} \mathrm{g}$ and $4^{\circ} \mathrm{C}$ for $3 \mathrm{~min}$ and the supernatant was discarded. The cells were re-suspended in $1 \mathrm{X}$ PBS at a density of $5 \times 10^{5}-1 \times 10^{6}$ cells $/ \mathrm{ml}$. A total of $500 \mu \mathrm{l}$ of the sample solution was transferred to a 5-ml culture tube and incubated with $5 \mu \mathrm{l}$ of FITC-conjugated Annexin V (Nanjing KeyGen Biotech Co., Ltd., Nanjing, China) and $5 \mu \mathrm{l}$ of PI (Nanjing KeyGen Biotech Co., Ltd.) for $15 \mathrm{~min}$ at room temperature in the dark. A total volume of $300 \mu \mathrm{l}$ of 1X PBS was added to each sample tube, and the samples were analyzed with fluorescence activated cell sorting flow cytometers (Beckman Coulter, Inc., Brea, CA, USA) using CellQuest software (version 5.2.1; BD Biosciences, San Jose, CA, USA).

Western blot analysis. Cell proteins were extracted using lysis buffer (Beyotime Institute of Biotechnology, Shanghai, China) and incubated on ice for protein extraction. Protein concentration was measured using a BCA Protein Assay Kit (Beyotime Institute of Biotechnology). The protein extract $(40 \mu \mathrm{g})$, which was prepared from cells, was separated using $10 \%$ SDS-PAGE and transferred to a polyvinylidene fluoride membrane (EMD Millipore, Billerica, MA, USA). The membrane was blocked with $5 \%$ skimmed milk (in PBS; pH 7.2; containing $0.1 \%$ Tween-20) for $2 \mathrm{~h}$ at room temperature and incubated at $4{ }^{\circ} \mathrm{C}$ overnight with the primary antibodies, which was then followed by incubation with horseradish peroxidase-conjugated secondary antibody (1:50,000; cat. no. BA1054; Wuhan Boster Biological Technology., Ltd., Wuhan, China) for $2 \mathrm{~h}$ at room temperature. The primary antibodies used were rabbit polyclonal anti-GAPDH (1:1,000; cat. no. AB-P-R 001; Hangzhou Xianzhi Biotechnology Co., Ltd., Hangzhou, China), rabbit polyclonal anti-phosphorylated p70S6 kinase (p-p70S6K; 1:600; cat. no. ab2571; Abcam, Cambridge, UK), rabbit polyclonal anti-p70S6K (1:1,200; cat. no. 14485-1-AP; ProteinTech Group, Inc., Wuhan, China), rabbit monoclonal anti-p-Akt (1:1,000; cat. no. 13038S, CST, MA, USA), rabbit monoclonal anti-Akt (1:1,000; cat. no. 4691S; Cell Signaling Technology, Inc., Danvers, MA, USA), rabbit polyclonal anti-ezrin (1:600; cat. no. 26056-1-AP; ProteinTech Group, Inc.) and rabbit polyclonal anti- $\alpha$-smooth muscle actin (SMA;1:5,000; cat. no. 55135-1-AP; ProteinTech Group, Inc.). The immunoreactive proteins were visualized using ECL plus detection reagents (Thermo Fisher Scientific, Inc.). ImageJ software (version 1.48u; National Institutes of Health, Bethesda, MD, USA) was used for the densitometry analysis.

Immunofluorescence staining. Cells grown on glass cover slips were fixed with $4 \%$ paraformaldehyde at room temperature for 15 min and permeabilized with PBS containing 0.5\% Triton $\mathrm{X}-100$ for $5 \mathrm{~min}$. Following three washes with PBS, cells were incubated in a blocking buffer containing $3 \%$ bovine serum albumin (Wuhan Boster Biological Technology Co., Ltd., Wuhan, China) in PBS for $30 \mathrm{~min}$ at room temperature, followed by incubation with ezrin (ab75840) and $\alpha$-SMA (ab5694) primary antibodies (1:100; Abcam) overnight at $4^{\circ} \mathrm{C}$. Following this, the cells were stained with a secondary anti-body labeled with fluorescein (Alexa Fluor 488-conjugated AffiniPure anti-goat secondary antibody (BA1032); IgG-Cy3; 1:100; Wuhan Boster Biological Technology Co., Ltd.). Following counterstaining with 4,6-diamidino-2-phenylindole (Beyotime Institute of Biotechnology) for $5 \mathrm{~min}$ at room temperature, cells were washed again with PBS and coverslips were transferred onto glass slides. Cells were observed and imaged were captured (magnification, x400) using fluorescent microscopy (BX53; Olympus Corporation, Tokyo, Japan). Image J software was used for fluorescence signal analysis. All experiments were performed a minimum of three times. 
Statistical analysis. Statistical analysis was performed using GraphPad 6.0 software (GraphPad Software, Inc., La Jolla, CA, USA). All data were expressed as the mean \pm standard error of the mean and were representative of three independent experiments. Comparisons between multiple groups were performed using one-way analysis of variance followed by a Bonferroni post hoc multiple comparison test. $\mathrm{P}<0.05$ was considered to indicate a statistically significant difference.

\section{Results}

Inhibition of the mTOR signaling pathway suppresses $H G$-induced podocyte viability. As indicated in Fig. 1, HG significantly increased podocyte viability when compared with the $\mathrm{M}$ group. However, rapamycin treatment significantly suppressed podocyte viability when compared with the HG group. Notably, the dual mTORC1 and mTORC2 inhibitor, KU0063794, further suppressed HG-induced cell viability when compared with the HG + rapamycin group. These results indicates that the mTORC1 and mTORC2 signaling pathways may be involved in HG regulation of podocyte viability.

Inhibition of the mTOR signaling pathway suppresses HG-induced podocyte apoptosis. As indicated in Fig. 2, HG significantly increased podocyte apoptosis when compared with the $\mathrm{M}$ group. However, rapamycin treatment significantly suppressed the HG-induced effect when compared with the HG group. KU0063794 further suppressed the HG-induced effect on apoptosis when compared with the HG + rapamycin group. These results indicate that mTORC1 and mTORC2 signaling pathways may be involved in $\mathrm{HG}$ regulation of podocyte apoptosis.

Inhibition of mTORC2 signaling suppresses the effect of $H G$ on the expression of ezrin and $\alpha$-SMA. mTORC1 regulates various major processes, including protein and lipid synthesis, proliferation, apoptosis and autophagy (27). p70s6k is the downstream signaling protein of mTORC1. Generally, p70s6k has been regarded as the marker of the mTORC1 signaling pathway, and the change of p-p70s6k expression can indicate a change of mTORC1 activity (28). Notably, mTORC2 participates in the regulation of several members of the AGC subfamily of kinases, including protein kinase C- $\alpha$, serumand glucocorticoid-induced protein kinase 1 and Akt (27). In addition, Akt can regulate a series of cellular processes, including metabolism, survival, apoptosis, growth and proliferation, via the phosphorylation of several effectors (27). Akt is the downstream signaling protein of the $\mathrm{mTORC} 2$ signaling pathway (29). It the present study, a change of p-Akt expression was considered to indicate a change of mTORC2 activity. Thus, the activities of p70s6k and Akt were examined in podocytes. The present results indicated that the expression levels of p-p70s6k and p-Akt were significantly increased by HG when compared with the M group. Notably, rapamycin treatment significantly inhibited the HG-induced increase of p-p70s6k, but not p-Akt; however, KU0063794 significantly inhibited the HG-induced increase of p-p70s6k and p-Akt (Fig. 3). These results suggested that rapamycin significantly inhibited the mTORC1 signaling pathway and KU0063794 significantly inhibited the mTORC1 and mTORC1 signaling

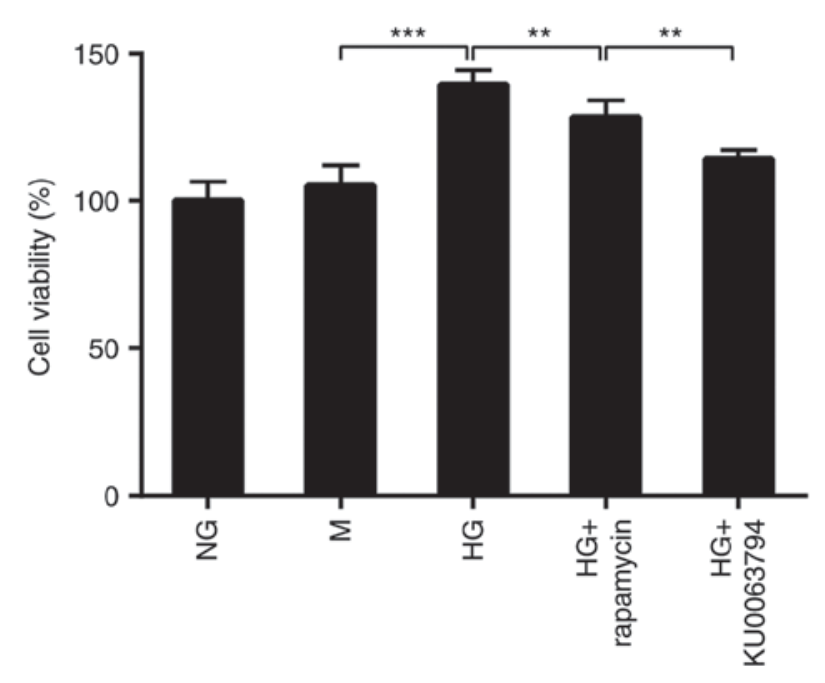

Figure 1. Cell viability in podocytes. Rapamycin significantly suppressed the HG-induced podocyte viability. KU0063794 further enhanced the suppression of viability. Data were presented as the mean \pm standard error of the mean and were representative of three independent experiments. ${ }^{* *} \mathrm{P}<0.01$ and ${ }^{* * *} \mathrm{P}<0.001$ as indicated. $\mathrm{HG}$, high glucose; $\mathrm{NG}$, normal glucose; $\mathrm{M}$, mannitol.

pathways. Ezrin and $\alpha$-SMA are cytoskeleton-associated proteins that serve a key role in cell surface structure adhesion, migration and organization $(30,31)$. In the present study, the expression of ezrin was significantly reduced by HG when compared with the $\mathrm{M}$ group, whereas the expression of $\alpha$-SMA was significantly increased, KU0063794, but not rapamycin, significantly suppressed the effect of $\mathrm{HG}$ on ezrin and $\alpha$-SMA expression levels (Fig. 3). Furthermore, intense fluorescence demonstrated ezrin was predominantly localized on the cell membrane and in cytoplasm (Fig. 4A). A total of $24 \mathrm{~h}$ following $\mathrm{HG}$ treatment, the fluorescence density of ezrin was significantly decreased when compared with the M group. KU0063794, but not rapamycin, significantly suppressed the effect of HG on ezrin expression levels. Notably, weak fluorescence indicated $\alpha$-SMA was also predominantly localized on the cell membrane and in the cytoplasm. A total of 24 following HG treatment, the fluorescence density of $\alpha$-SMA was significantly increased. Similarly, KU0063794, but not rapamycin, significantly suppressed the effect of HG on the $\alpha$-SMA expression levels (Fig. 4B). These results indicated that mTORC2 signaling, but not mTORC1 signaling, may primarily mediate the regulation of the cytoskeleton.

\section{Discussion}

Podocytes are highly specialized cells in the kidney, which can release growth factors for mesangial and endothelial cells, and can regulate glomerular filtration (32). Injury or loss to podocytes has a crucial role in the regulation of the pathological process of proteinuric kidney disease, including DN (33-35). Apoptosis and the functional loss of podocytes contribute to the development of DN (36). Previous studies have indicated that podocyte injury is associated with a reduced level of autophagy (37-39) and an increased level of inflammation $(40,41)$ and apoptosis $(23,42)$ during exposure to HG conditions. The present study revealed increased viability 

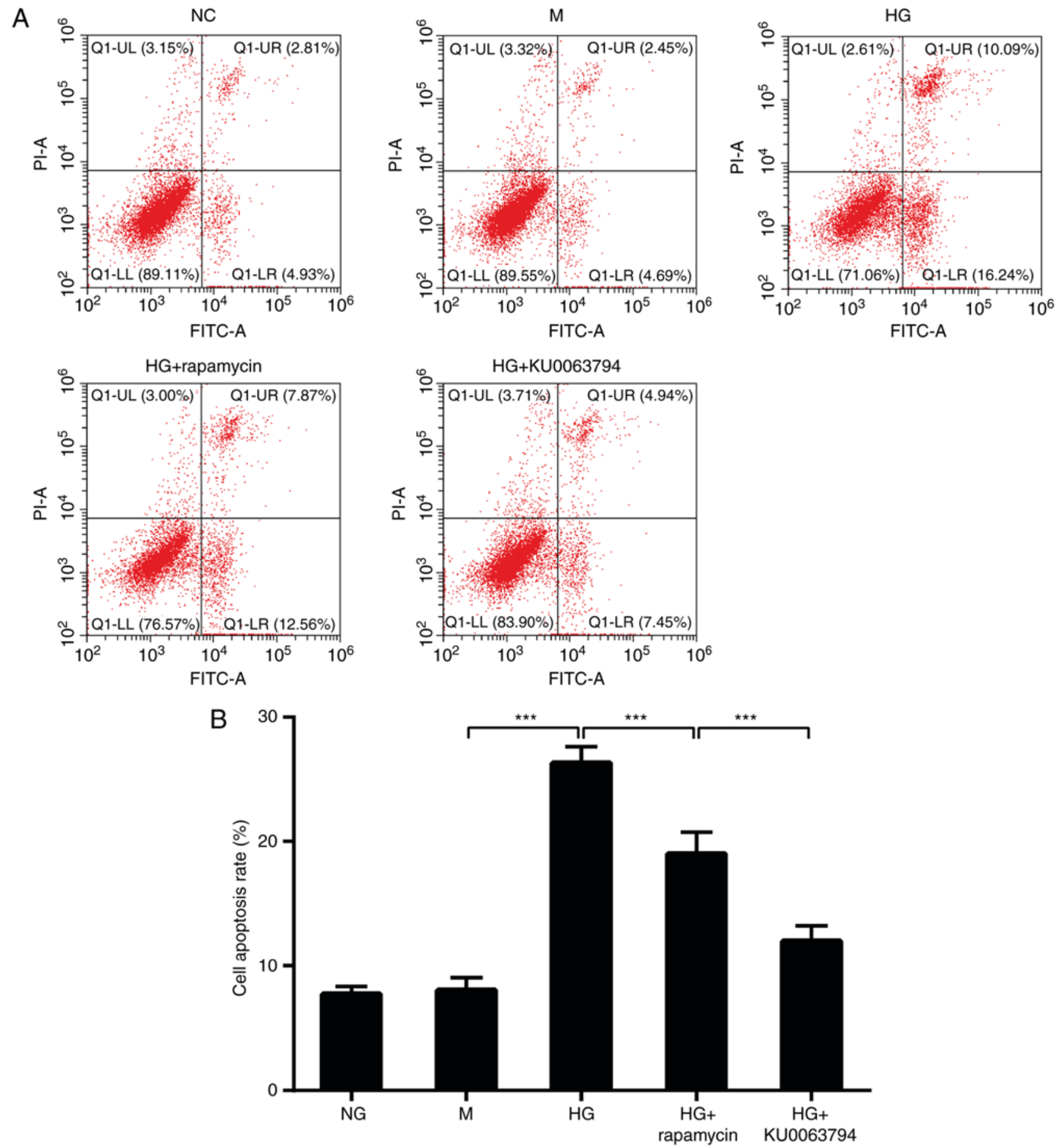

Figure 2. Cell apoptosis in podocytes. (A and B) Rapamycin significantly suppressed the HG-induced podocyte apoptosis. KU0063794 further enhanced the suppression of apoptosis. Data were presented as the mean \pm standard error of the mean and were representative of three independent experiments. ${ }^{* * *} \mathrm{P}<0.001$ as indicated. HG, high glucose; NG, normal glucose; M, mannitol; FITC, fluorescein isothiocyanate.

and apoptosis in podocytes $24 \mathrm{~h}$ following $\mathrm{HG}$ exposure. Furthermore, inhibition of mTORC1 by rapamycin significantly attenuated the HG-induced viability and apoptosis, and dual mTORC1 and mTORC2 inhibition by KU0063794 further attenuated the HG effect. In addition, KU0063794, but not rapamycin, was indicated to inhibit the effects of HG on the expression levels of ezrin and $\alpha$-SMA.

The mTOR signaling pathway has important roles in regulating cell metabolism, growth, proliferation and apoptosis $(19,43)$. Pharmacological inhibition of mTOR and selective targeting of mTORC1 or mTORC2 in podocytes has been suggested to elucidate their role in renal cell homeostasis (44). It has been reported that podocyte-specific embryonic knockout of mTORC1 in mice resulted in early albuminuria, later development of glomerulosclerosis, loss of weight and increase of mortality (45). Mice with podocyte-specific loss of mTORC2 did not demonstrate significant phenotypic differences when compared with the littermate controls, with the exception of transient albuminuria following protein overload in combination with the deletion of both mTOR complexes from podocytes, 

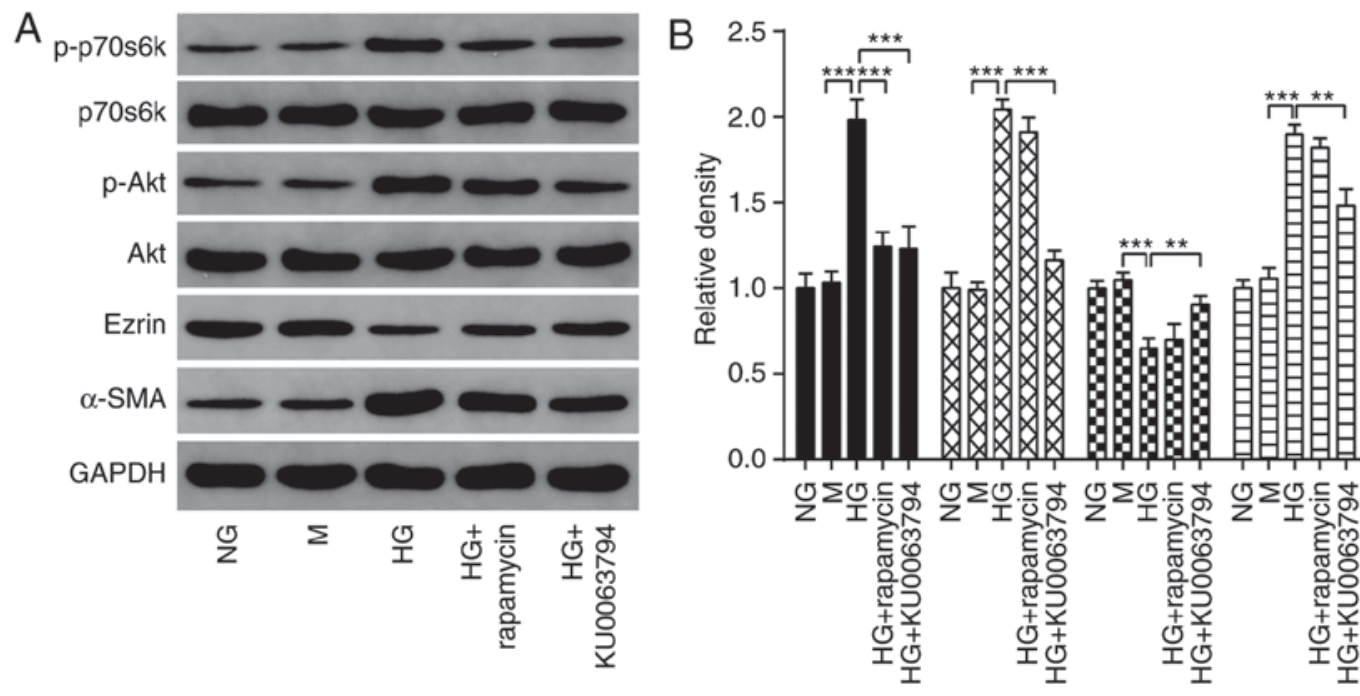

$\mathrm{p}-\mathrm{p} 70 \mathrm{~s} 6 \mathrm{k} / \mathrm{p} 70 \mathrm{~s} 6 \mathrm{k}$

区 p-Akt/Akt

$\mathbf{B}$ Ezrin/GAPDH

曰 $\alpha$-SMA/GAPDH

Figure 3. Expression of mammalian target of rapamycin signaling-associated proteins and cytoskeleton-associated proteins in podocytes. (A) Representative image of western blot analysis. (B) Quantitative graph of the protein expression levels. Data were presented as the mean \pm standard error of the mean and were representative of three independent experiments. Rapamycin significantly suppressed the HG-induced activation of p70s6k, but not Akt. KU0063794 significantly suppressed the HG-induced activation of p70s6k and Akt. HG significantly reduced the expression of ezrin and increased the expression of $\alpha$-SMA. However, KU0063794, but not rapamycin, significantly inhibited the effect of HG on the expression of ezrin and $\alpha$-SMA. ${ }^{* *} \mathrm{P}<0.01$ and ${ }^{* * * *} \mathrm{P}<0.001$ as indicated. HG, high glucose; NG, normal glucose; M, mannitol; p-, phosphorylated; $\alpha$-SMA, $\alpha$-smooth muscle actin.

A
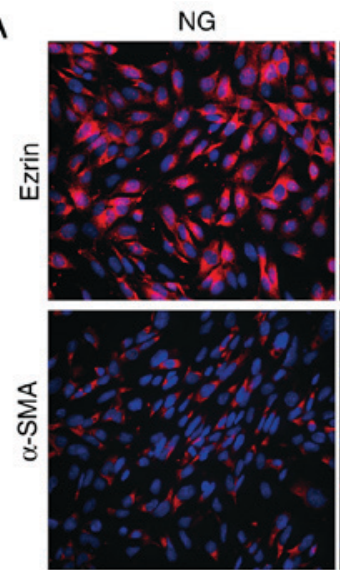
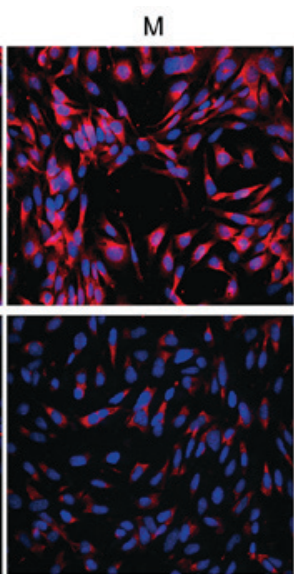

B
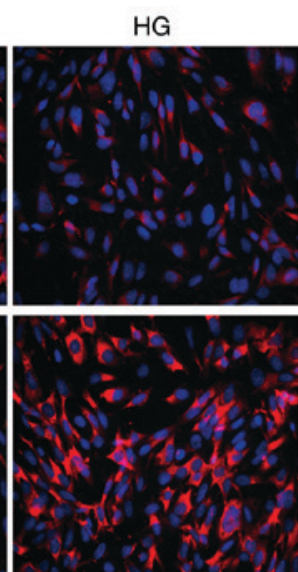

$\mathrm{HG}+$ rapamycin
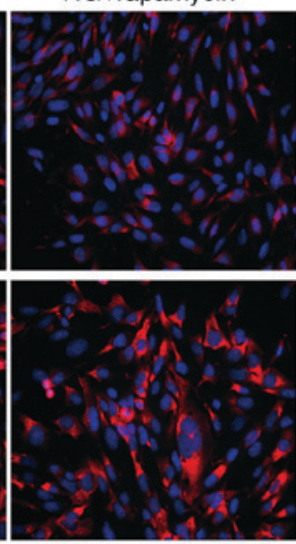

$\mathrm{HG}+\mathrm{KU} 0063794$
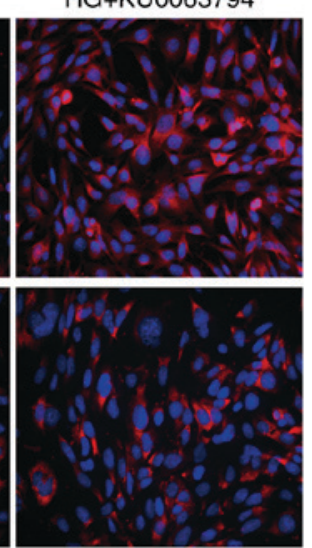

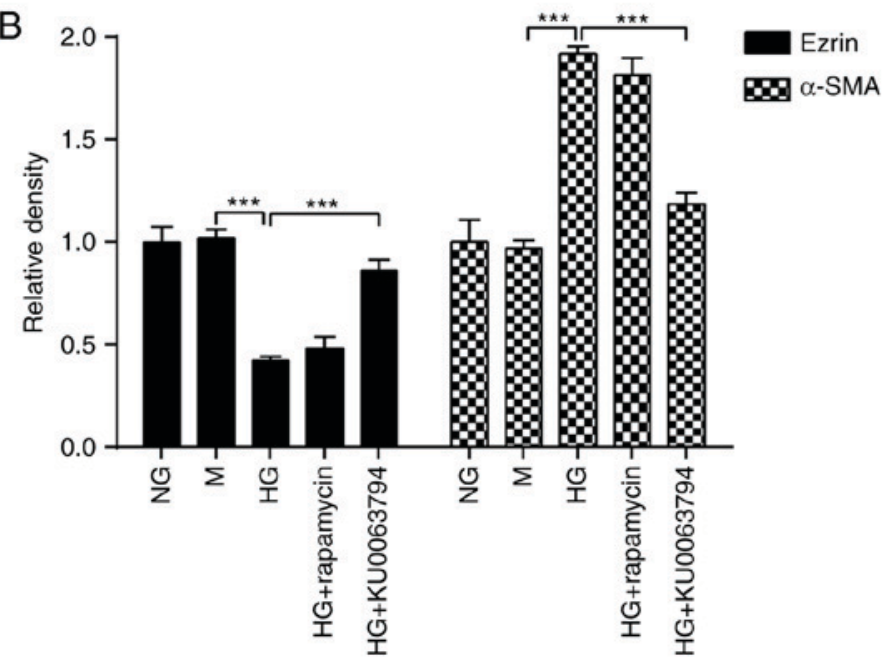

Figure 4. Immunofluorescence staining of cytoskeleton-associated proteins in podocytes. (A) Ezrin and $\alpha$-SMA were primarily localized on the cell membrane and in the cytoplasm. Ezrin- and $\alpha$-SMA-positive cells were stained with red fluorescence (magnification, x400). (B) Quantitative graph of the fluorescence density of ezrin- and $\alpha$-SMA-positive cells. Data were presented as the mean \pm standard error of the mean and were representative of three independent experiments. HG significantly reduced the fluorescence density of ezrin-positive cells and significantly increased the fluorescence density of $\alpha$-SMA-positive cells. KU0063794, but not rapamycin, significantly inhibited the effect of HG on the fluorescence density of ezrin- and $\alpha$-SMA-positive cells. ${ }^{* * *}$ P<0.001 as indicated. HG, high glucose; NG, normal glucose; M, mannitol; p-, phosphorylated; $\alpha$-SMA, $\alpha$-smooth muscle actin. 
which caused an early (at 6 weeks old) fulminant proteinuric phenotype (44). These previous findings indicated that there may be some degree of interaction between mTORC1 and mTORC2 in regulating the development and homeostasis of podocytes. Over activation of mTORC1 can also lead to severe pathologic effects, including hallmarks of DN (46). A previous study revealed that inhibition of $\mathrm{mTORC} 1$ signaling activity prevented glomerulosclerosis and significantly ameliorates the progression of glomerular disease in DN (47), and inhibition of mTORC2 signaling activity reduced $\mathrm{HG}$-induced podocyte apoptosis and attenuated albuminuria (48). Notably, previous findings have suggested that the activation of mTOR signaling is involved in HG-induced podocyte injury (23). It has been reported that the activated mTOR signaling can result in energy consumption, which in turn induces apoptosis in HG-treated podocytes (23). Furthermore, a previous study demonstrated that suppression of mTOR signaling protects against HG-induced apoptosis in $\beta$ cells (49), indicating that mTOR signaling may serve crucial roles in the regulation of $\mathrm{HG}$-induced apoptosis. In the present study, it was indicated that HG induced podocyte injury, which was reflected by the results on podocyte apoptosis, and altered the expression levels of cytoskeleton-associated proteins. However, the inhibition of mTORC1 activity significantly attenuated the $\mathrm{HG}$ effects, and dual mTORC1 and mTORC2 inhibition further attenuated the $\mathrm{HG}$ effects. These findings indicate that the mTORC1 and mTORC2 signaling pathways may be involved in HG-induced podocyte injury. As the expression levels of p-p70s6k and p-Akt can indicate the activation of mTORC1 and the mTORC2 signaling pathway, respectively (27), the expression levels of p-p70s6k and p-Akt were examined in podocytes in the present study. The following was indicated in the present study: HG significantly increased the p-p70s6k and p-Akt expression levels; rapamycin and KU0063794 significantly inhibited the increase in HG-induced p-p70s6k expression levels; rapamycin inhibited the increase in HG-induced p-Akt expression levels; and KU0063794 significantly inhibited the increase in the HG-induced p-Akt expression levels. These findings suggest that $\mathrm{HG}$ could significantly induce the activation of mTOR signaling pathway and that this may be suppressed by rapamycin or KU0063794.

Ezrin act as a linker between the cell membrane and the actin cytoskeleton. This linker function provides ezrin essential roles in various fundamental cellular processes, including cell adhesion, motility, cytokinesis, phagocytosis, determination of cell shape, polarity and surface structure, and integration of membrane transport with signaling pathways $(50,51)$. It has been reported that abnormal expression of ezrin and actin initiates the morphological changes of arsenic-induced apoptosis in human esophageal epithelial cells (52). Ezrin is downregulated in glomeruli of patients with DN, and has been identified to serve a role in regulating the development of renal complications in diabetes through transport of glucose and organization of the actin cytoskeleton in podocytes (53). It has been reported that Ethanolic Ginkgo biloba leaf extract prevents renal fibrosis via mTOR signaling in DN (54), indicating that mTOR signaling may be involved in the regulation of cytoskeletal proteins. In the present study, it was revealed that HG significantly reduced the expression levels of ezrin, and KU0063794, but not rapamycin, suppressed the effect of HG on the ezrin expression levels. This finding suggested that the cytoskeleton-associated proteins may be primarily regulated by mTORC2 (and not the mTORC1) signaling pathway. Notably, $\alpha$-SMA is the actin isoform that serves an important role in fibrogenesis (55). A previous study indicated that the $\alpha$-SMA expression in the renal tubulointerstitium was highly upregulated in diabetic rats when compared with the non-diabetic rats (56). In the present study, it was revealed that HG significantly increased the expression of $\alpha$-SMA, and KU0063794, but not rapamycin, significantly suppressed the effect of $\mathrm{HG}$ on $\alpha$-SMA expression. This result further supported that the cytoskeleton-associated proteins may be primarily regulated by the mTORC 2 (and not the mTORC1) signaling pathway. A previous study has reported that disruption of the podocyte cytoskeleton can induce podocyte apoptosis (57), indicating that cytoskeleton-associated proteins may serve important roles in regulating podocyte apoptosis. In the present study, it was demonstrated that HG induced podocyte apoptosis, which was accompanied by the disruption of cytoskeleton, including the decreased expression of ezrin and the increased expression of $\alpha$-SMA. These results suggest that the altered expression of cytoskeleton-associated proteins in HG-treated podocytes may promote the development of apoptosis.

According to the present results, rapamycin significantly inhibited the increase of p-p70s6k, but not p-Akt, in HG-treated podocytes, indicating that rapamycin could significantly block mTORC1 activity. Rapamycin did not significantly suppress the effect of $\mathrm{HG}$ on the expression levels of ezrin and $\alpha$-SMA, which suggested that the mTORC1 pathway may not be involved in the regulation of cytoskeletal proteins in podocytes. Figs. 1 and 2 demonstrate that mTORC1 and mTORC2 were involved in the regulation of cell viability and apoptosis in HG-treated podocytes. Previous studies have indicated that cytoskeletal proteins are associated with cell viability and apoptosis $(52,58)$. The present results suggest that the role of the mTORC1 signaling pathway on cell viability and apoptosis may not be through the regulation of cytoskeletal proteins. During the development of DN, the formation of advanced glycation end products (AGEs) were significantly enhanced in the presence of HG levels (59). It has been reported that AGEs interact with the N-terminal domain of ezrin and inhibit its actions in proximal tubule cells (60). Notably, a previous study indicated that AGE treatment reduced podocyte adhesion to fibronectin and inhibited migration; however, ezrin overexpression completely reversed the AGE inhibition of podocyte adhesion to fibronectin and partially reversed AGE-induced inhibition of migration (61). This suggests that HG-induced podocyte injury may be mediated by an increased level of AGEs. Further in-depth study on the role of AGEs on podocytes is required.

In conclusion, the present study demonstrated that HG induced effects on podocytes, including increased podocyte apoptosis and changes in the expression levels of cytoskeleton-associated proteins $24 \mathrm{~h}$ following $\mathrm{HG}$ treatment. Notably, the cell viability was also increased following $\mathrm{HG}$ treatment. This suggests that with prolongation of the time of HG action, the cell viability may be reduced due to the increased level of apoptosis. These findings indicated that mTORC1 and mTORC2 signaling pathways may be involved in the regulation of podocyte viability and apoptosis, and that the mTORC2 signaling pathway may be the primary signaling 
pathway involved in the regulation of cytoskeleton-associated proteins.

\section{Acknowledgements}

Not applicable.

\section{Funding}

This study was supported by the Natural Science Foundation of Jiangxi Province (grant no. 20161BAB205233).

\section{Availability of data and materials}

All data generated or analyzed during this study are included in this published article.

\section{Authors' contributions}

QL, YZ and QJ performed the experiments. QL and JZ participated in data collection and drafted the manuscript. QL and $\mathrm{CW}$ performed the statistical analysis and designed the present study. All authors read and approved the final manuscript.

\section{Ethics approval and consent to participate}

Not applicable.

\section{Patient consent for publication}

Not applicable.

\section{Competing interests}

The authors declare that they have no competing interests.

\section{References}

1. Lippert J, Ritz E, Schwarzbeck A and Schneider P: The rising tide of endstage renal failure from diabetic nephropathy type II-an epidemiological analysis. Nephrol Dial Transplant 10: 462-467, 1995.

2. Graves DT and Kayal RA: Diabetic complications and dysregulated innate immunity. Front Biosci 13: 1227-1239, 2008.

3. Ziyadeh FN: Mediators of diabetic renal disease: The case for tgf-Beta as the major mediator. J Am Soc Nephrol 15 (Suppl 1): S55, 2004.

4. Yasuda-Yamahara M, Kume S, Tagawa A, Maegawa $\mathrm{H}$ and Uzu T: Emerging role of podocyte autophagy in the progression of diabetic nephropathy. Autophagy 11: 2385-2386, 2015.

5. Jefferson JA, Shankland SJ and Pichler RH: Proteinuria in diabetic kidney disease: A mechanistic viewpoint. Kidney Int 74: 22-36, 2008.

6. Mathieson PW: The podocyte as a target for therapies-new and old. Nat Rev Nephrol 8: 52-56, 2011.

7. Covington MD and Schnellmann RG: Chronic high glucose downregulates mitochondrial calpain 10 and contributes to renal cell death and diabetes-induced renal injury. Kidney Int 81: 391-400, 2012

8. Fox CS, Matsushita K, Woodward M, Bilo HJ, Chalmers J, Heerspink HJ, Lee BJ, Perkins RM, Rossing P, Sairenchi T, et al: Associations of kidney disease measures with mortality and end-stage renal disease in individuals with and without hypertension: A meta-analysis. Lancet 380: 1662-1673, 2012.

9. Li C and Siragy HM: High glucose induces podocyte injury via enhanced (pro)renin receptor-Wnt- $\beta$-catenin-snail signaling pathway. PLoS One 9: e89233, 2014.
10. Tryggvason K, Patrakka J and Wartiovaara J: Hereditary proteinuria syndromes and mechanisms of proteinuria. N Engl J Med 354: 1387-1401, 2006.

11. Coimbra TM, Janssen U, Gröne HJ, Ostendorf T, Kunter U, Schmidt H, Brabant $\mathrm{G}$ and Floege J: Early events leading to renal injury in obese Zucker (fatty) rats with type II diabetes. Kidney Int 57: 167-182, 2000.

12. Siu B, Saha J, Smoyer WE, Sullivan KA and Brosius FC: Reduction in podocyte density as a pathologic feature in early diabetic nephropathy in rodents: Prevention by lipoic acid treatment. Bmc Nephrol 7: 6, 2006.

13. Torres VE, Boletta A, Chapman A, Gattone V, Pei Y, Qian Q, Wallace DP, Weimbs T and Wüthrich RP: Prospects for mTOR inhibitor use in patients with polycystic kidney disease and hamartomatous diseases. Clin J Am Soc Nephrol 5: 1312-1329, 2010.

14. Wullschleger S, Loewith $\mathrm{R}$ and Hall MN: TOR signaling in growth and metabolism. Cell 124: 471-484, 2006.

15. Sarbassov DD, Ali SM, Kim DH, Guertin DA, Latek RR, Erdjument-Bromage H, Tempst P and Sabatini DM: Rictor, a novel binding partner of mTOR, defines a rapamycin-insensitive and raptor-independent pathway that regulates the cytoskeleton. Curr Biol 14: 1296-1302, 2004.

16. Mori S, Nada S, Kimura H, Tajima S, Takahashi Y, Kitamura A, Oneyama C and Okada M: The mTOR pathway controls cell proliferation by regulating the FoxO3a transcription factor via SGK1 kinase. PLoS One 9: e88891, 2014.

17. Tsai TF, Lin JF, Chou KY, Lin YC, Chen HE and Hwang TI: miR-99a-5p acts as tumor suppressor via targeting to mTOR and enhances RAD001-induced apoptosis in human urinary bladder urothelial carcinoma cells. Onco Targets Ther 11: 239-252, 2018.

18. Shortt J, Martin BP, Newbold A, Hannan KM, Devlin JR, Baker AJ, Ralli R, Cullinane C, Schmitt CA, Reimann M, et al: Combined inhibition of PI3K-related DNA damage response kinases and mTORC1 induces apoptosis in MYC-driven B-cell lymphomas. Blood 121: 2964-2974, 2013.

19. Hagiwara A, Nishiyama M and Ishizaki S: Branched-chain amino acids prevent insulin-induced hepatic tumor cell proliferation by inducing apoptosis through mTORC1 and mTORC2-dependent mechanisms. J Cell Physiol 227: 2097-2105, 2012.

20. Gurusamy N, Lekli I, Mukherjee S, Ray D, Ahsan MK, Gherghiceanu M, Popescu LM and Das DK: Cardioprotection by resveratrol: A novel mechanism via autophagy involving the mTORC2 pathway. Cardiovasc Res 86: 103-112, 2010.

21. Zhang HT, Wang WW, Ren LH, Zhao XX, Wang ZH, Zhuang DL and Bai YN: The mTORC2/Akt/NFKB pathway-mediated activation of TRPC6 participates in adriamycin-induced podocyte apoptosis. Cell Physiol Biochem 40: 1079-1093, 2016.

22. Cao W, Li M, Wu T, Feng F, Feng T, Xu Y and Sun C: $\alpha \mathrm{MSH}$ prevents ROS-induced apoptosis by inhibiting Foxo1/mTORC2 in mice adipose tissue. Oncotarget 8: 40872-40884, 2017.

23. Lei J, Zhao L, Zhang Y, Wu Y and Liu Y: High glucose-induced podocyte injury involves activation of mammalian target of rapamycin (mTOR)-induced endoplasmic reticulum (ER) stress. Cell Physiol Biochem 45: 2431-2443, 2018.

24. Altman JK, Szilard A, Goussetis DJ, Sassano A, Colamonici M, Gounaris E, Frankfurt O, Giles FJ, Eklund EA, Beauchamp EM and Platanias LC: Autophagy is a survival mechanism of acute myelogenous leukemia precursors during dual mTORC2/mTORC1 targeting. Clin Cancer Res 20: 2400-2409, 2014.

25. Hettmer S, Liu J, MillerCM,Lindsay MC, Sparks CA, Guertin DA, Bronson RT, Langenau DM and Wagers AJ: Sarcomas induced in discrete subsets of prospectively isolated skeletal muscle cells. Proc Natl Acad Sci USA 108: 20002-20007, 2011.

26. Zhang H, Berel D, Wang Y, Li P, Bhowmick NA, Figlin RA and Kim HL: A comparison of Ku0063794, a dual mTORC1 and mTORC2 inhibitor, and temsirolimus in preclinical renal cell carcinoma models. PLoS One 8: e54918, 2013.

27. Laplante M and Sabatini DM: mTOR signaling in growth control and disease. Cell 149: 274-293, 2012.

28. Boehlke C, Kotsis F, Patel V, Braeg S, Voelker H, Bredt S, Beyer T, Janusch H, Hamann C, Gödel M, et al: Primary cilia regulate mTORC1 activity and cell size through Lkb1. Nat Cell Biol 12: 1115-1122, 2010.

29. Lou JS, Xia YT, Wang HY, Kong XP, Yao P, Dong TTX, Zhou ZY and Tsim KWK: The WT1/MVP-mediated stabilization on mTOR/AKT axis enhances the effects of cisplatin in non-small cell lung cancer by a reformulated yu ping feng san herbal preparation. Front Pharmacol 9: 853, 2018. 
30. Arpin M, Chirivino D, Naba A and Zwaenepoel I: Emerging role for ERM proteins in cell adhesion and migration. Cell Adh Migr 5: 199-206, 2011.

31. Zhong L, Jin J, Zheng D, Guan W, Guo Y, Chen A, Peng Y, Gao Q, Zheng Y and Huang H: Influence of super-hydrophobic silicone rubber substrate on the growth and differentiation of human lens epithelial cells. J Mater Sci Mater Med 29: 176, 2018.

32. Dalla Vestra M, Masiero A, Roiter AM, Saller A, Crepaldi G and Fioretto P: Is podocyte injury relevant in diabetic nephropathy? Studies in patients with type 2 diabetes. Diabetes 52: 1031-1035, 2003.

33. Jauregui A, Mintz DH, Mundel P and Fornoni A: Role of altered insulin signaling pathways in the pathogenesis of podocyte malfunction and microalbuminuria. Curr Opin Nephrol Hypertens 18: 539-545, 2009.

34. Meyer TW, Bennett PH and Nelson RG: Podocyte number predicts long-term urinary albumin excretion in Pima Indians with Type II diabetes and microalbuminuria. Diabetologia 42 : 1341-1344, 1999.

35. Zhang C, Hou B, Yu S, Chen Q, Zhang N and Li H: HGF alleviates high glucose-induced injury in podocytes by GSK3 $\beta$ inhibition and autophagy restoration. Biochim Biophys Acta 1863: 2690-2699, 2016

36. Tung CW, Hsu YC, Shih YH, Chang PJ and Lin CL: Glomerular mesangial cell and podocyte injuries in diabetic nephropathy. Nephrology (Carlton) 23 (Suppl 4): S32-S37, 2018.

37. Xu L, Fan Q, Wang X, Li L, Lu X, Yue Y, Cao X, Liu J, Zhao X and Wang L: Ursolic acid improves podocyte injury caused by high glucose. Nephrol Dial Transplant 32: 1285-1293, 2017.

38. Sun J, Li ZP, Zhang RQ and Zhang HM: Repression of miR-217 protects against high glucose-induced podocyte injury and insulin resistance by restoring PTEN-mediated autophagy pathway. Biochem Biophys Res Commun 483: 318-324, 2017.

39. Miaomiao W, Chunhua L, Xiaochen Z, Xiaoniao C, Hongli L and Zhuo Y: Autophagy is involved in regulating VEGF during high-glucose-induced podocyte injury. Mol Biosyst 12: 2202-2212, 2016.

40. Wei M, Li Z, Xiao L and Yang Z: Effects of ROS-relative NF-кB signaling on high glucose-induced TLR4 and MCP-1 expression in podocyte injury. Mol Immunol 68: 261-271, 2015.

41. Li J, Wang B, Zhou G, Yan X and Zhang Y: Tetrahydroxy stilbene glucoside alleviates high glucose-induced MPC5 podocytes injury through suppression of NLRP3 inflammasome. Am J Med Sci 355: 588-596, 2018

42. Shen ZN, Gan H, Du X and Wang X: Effect of PFT- $\alpha$ on ubiquitin and apoptosis of podocyte injured by high glucose. J Chongqing Med Univ 4: 412-415, 2011.

43. Huo HZ, Zhou ZY, Wang B, Qin J, Liu WY and Gu Y: Dramatic suppression of colorectal cancer cell growth by the dual mTORC1 and mTORC2 inhibitor AZD-2014. Biochem Biophys Res Commun 443: 406-412, 2014

44. Fantus D, Rogers NM, GrahammerF, Huber TB and Thomson AW: Roles of mTOR complexes in the kidney: Implications for renal disease and transplantation. Nat Rev Nephrol 12: 587-609, 2016.

45. Gödel M, Hartleben B, Herbach N, Liu S, Zschiedrich S, Lu S, Debreczeni-Mór A, Lindenmeyer MT, Rastaldi MP, Hartleben $\mathrm{G}$, et al: Role of mTOR in podocyte function and diabetic nephropathy in humans and mice. J Clin Invest 121: 2197-2209, 2011

46. Inoki K, Mori H, Wang J, Suzuki T, Hong S, Yoshida S, Blattner SM, Ikenoue T, Rüegg MA, Hall MN, et al: mTORC1 activation in podocytes is a critical step in the development of diabetic nephropathy in mice. J Clin Invest 121: 2181-2196, 2011

47. Gödel M, Hartleben B, Herbach N, Liu S, Zschiedrich S, Lu S, Debreczeni-Mór A, Lindenmeyer MT, Rastaldi MP, Hartleben $\mathrm{G}$, et al: Role of mTOR in podocyte function and diabetic nephropathy in humans and mice. J Clin Invest 121 2197-2209, 2011
48. Eid S, Boutary S, Braych K, Sabra R, Massaad C, Hamdy A Rashid A, Moodad S, Block K, Gorin Y, et al: mTORC2 signaling regulates nox4-induced podocyte depletion in diabetes. Antioxid Redox Signal 25: 703-719, 2016.

49. Yang Z, Liu F, Qu H, Wang H, Xiao X and Deng H: 1, 25(OH)2D3 protects $\beta$ cell against high glucose-induced apoptosis through mTOR suppressing. Mol Cell Endocrinol 414: 111-119, 2015.

50. Ng T, Parsons M, Hughes WE, Monypenny J, Zicha D, Gautreau A, Arpin M, Gschmeissner S, Verveer PJ, Bastiaens PI and Parker PJ: Ezrin is a downstream effector of trafficking PKC-integrin complexes involved in the control of cell motility. EMBO J 20: 2723-2741, 2001.

51. Bretscher A, Edwards $\mathrm{K}$ and Fehon RG: ERM proteins and merlin: Integrators at the cell cortex. Nat Rev Mol Cell Biol 3: 586-599, 2002.

52. Shen ZY, Xu LY, Li EM, Li JT, Chen MH, Shen J and Zeng Y: Ezrin, actin and cytoskeleton in apoptosis of esophageal epithelial cells induced by arsenic trioxide. Int J Mol Med 12: 341-347, 2003.

53. Wasik AA, Koskelainen S, Hyvönen ME, Musante L, Lehtonen E, Koskenniemi K, Tienari J, Vaheri A, Kerjaschki D, Szalay C, et al: Ezrin is down-regulated in diabetic kidney glomeruli and regulates actin reorganization and glucose uptake via GLUT1 in cultured podocytes. Am J Pathol 184: 1727-1739, 2014.

54. Lu Q, Zuo WZ, Ji XJ, Zhou YX, Liu YQ, Yao XQ, Zhou XY, Liu YW, Zhang F and Yin XX: Ethanolic Ginkgo biloba leaf extract prevents renal fibrosis through $\mathrm{Akt} / \mathrm{mTOR}$ signaling in diabetic nephropathy. Phytomedicine 22: 1071-1078, 2015.

55. Kawasaki Y, Imaizumi T, Matsuura H, Ohara S, Takano K, Suyama K, Hashimoto K, Nozawa R, Suzuki H and Hosoya M: Renal expression of alpha-smooth muscle actin and c-Met in children with Henoch-Schönlein purpura nephritis. Pediatr Nephrol 23: 913-919, 2008.

56. Ren X, Guan G, Liu G and Liu G: Irbesartan ameliorates diabetic nephropathy by reducing the expression of connective tissue growth factor and alpha-smooth-muscle actin in the tubulointerstitium of diabetic rats. Pharmacology 83: 80-87, 2009.

57. Huang Z, Zhang L, Chen Y, Zhang H, Yu C, Zhou F, Zhang Z, Jiang L, Li R, Ma J, et al: RhoA deficiency disrupts podocyte cytoskeleton and induces podocyte apoptosis by inhibiting YAP/dendrin signal. BMC Nephrol 17: 66, 2016.

58. Yao Q, Pei Y, Zhang X and Xie B: microRNA-96 acts as a tumor suppressor gene in human osteosarcoma via target regulation of EZRIN. Life Sci 203: 1-11, 2018

59. Yan SJ, Wang L, Li Z, Zhu DN, Guo SC, Xin WF, Yang YF, Cong X, Ma T, Shen PP, et al: Inhibition of advanced glycation end product formation by $\mathrm{Pu}$-erh tea ameliorates progression of experimental diabetic nephropathy. J Agric Food Chem 60: 4102-4110, 2012

60. Mcrobert EA, Gallicchio M, Jerums G, Cooper ME and Bach LA: The amino-terminal domains of the ezrin, radixin, and moesin (ERM) proteins bind advanced glycation end products, an interaction that may play a role in the development of diabetic complications. J Biol Chem 278: 25783-25789, 2003

61. McRobert EA and Bach LA: Ezrin contributes to impaired podocyte migration and adhesion caused by advanced glycation end products. Nephrology (Carlton) 21: 13-20, 2016.

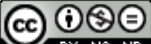

This work is licensed under a Creative Commons Attribution-NonCommercial-NoDerivatives 4.0 International (CC BY-NC-ND 4.0) License. 